\title{
Total Synthesis of (-)-Barbatusol, (+)-Demethylsalvicanol, $(-)$-Brussonol, and (+)-Grandione
}

\author{
George Majetich* and Ge Zou \\ Department of Chemistry, University of Georgia, Athens, Georgia 30602 \\ majetich@chem.uga.edu
}

\begin{abstract}
SUPPORTING INFORMATION
Table of Contents S 1

General Procedures S1

Experimental Procedures S1-S5
\end{abstract}

General Procedures: All reactions were run under a nitrogen atmosphere and monitored by TLC analysis. Unless otherwise indicated, all ethereal workups consisted of the following procedure: The organic solvent was removed under reduced pressure on a rotary evaporator, and the residue was taken up in diethyl ether, washed with brine, and dried over anhydrous magnesium sulfate. Filtration, followed by concentration at reduced pressure on a rotary evaporator and at 1 torr to a constant weight, afforded a crude residue which was purified by flash chromatography using silica gel 60 (230-400 mesh ASTM) and distilled reagent grade petroleum ether and diethyl ether. Melting points were recorded on a Laboratory Devices Mel-Temp 3.0. ${ }^{1} \mathrm{H}$ and ${ }^{13} \mathrm{C}$ NMR spectra were recorded on Bruker AVB-400 and DRX-500 MHz spectrometers with ${ }^{13} \mathrm{C}$ operating frequencies of $100 \mathrm{MHz}$ and $125 \mathrm{MHz}$, respectively. Proton NMR spectra were obtained in $\mathrm{CDCl}_{3}$ and were calibrated using trace $\mathrm{CHCl}_{3}$ present $(\delta 7.27)$ as an internal reference. Carbon NMR spectra were obtained in $\mathrm{CDCl}_{3}$ and were calibrated using trace $\mathrm{CHCl}_{3}$ present $(\delta 77.23)$ as an internal reference. The IR spectra were obtained using an Avatar 360FT-IR and are reported in frequency of absorption $\left(\mathrm{cm}^{-1}\right)$. Only selected IR absorbencies are reported. High resolution MS were taken using a LCT Premier from Waters.

\section{Experimental Procedures:}

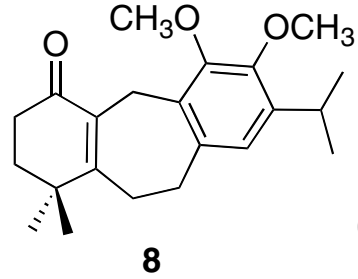
(S)-MeCBS $\mathrm{BH}_{3}$
THF, rt $(90 \%, 96 \%$ ee)

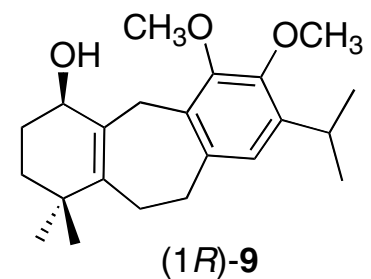

$(1 R)-9$

To a solution of $8(2.26 \mathrm{~g}, 6.60 \mathrm{mmol})$ in $80 \mathrm{~mL}$ of anhydrous THF, $\mathrm{BH}_{3}-\mathrm{Me}_{2} \mathrm{~S}(1.3 \mathrm{~mL}, 1 \mathrm{M}, 1.32 \mathrm{mmol})$ was added. After the mixture was stirred for a 15 min-period at $40{ }^{\circ} \mathrm{C},(S)-\mathrm{CBS}(0.65 \mathrm{~mL}, 10 \sim 10.2 \mathrm{M})$ in 30 
$\mathrm{mL}$ of THF was added using a syringe pump over an 8-h period. The reaction mixture was cooled to $0{ }^{\circ} \mathrm{C}$ and methanol was added dropwise. The mixture was then concentrated and purified by silica gel column chromatography (elution with EtOAc/pet ether $=1: 4)$ to give $2.05 \mathrm{~g}(90 \%$ yield, $>96 \% \quad e e)$ of allylic alcohol $(1 R)-9\left[\mathrm{R}_{\mathrm{f}}=0.17\right.$, EtOAc/pet ether $\left.=1: 8\right]: \quad[\alpha]_{\mathrm{D}}=+20.88\left(\mathrm{CHCl}_{3} ; c 0.050\right) ;{ }^{1} \mathrm{H} \mathrm{NMR}\left(500 \mathrm{MHz}, \mathrm{CDCl}_{3}\right) \delta$ $6.91(\mathrm{~s}, 1 \mathrm{H}), 4.06(\mathrm{~m}, 1 \mathrm{H}), 3.84(\mathrm{~s}, 3 \mathrm{H}), 3.86(\mathrm{~s}, 3 \mathrm{H}), 3.61(\mathrm{~m}, 1 \mathrm{H}), 3.27$ (septet, $J=7.0 \mathrm{~Hz}, 1 \mathrm{H}), 3.01-2.84(\mathrm{~m}$, $2 \mathrm{H}), 2.51-2.39(\mathrm{~m}, 2 \mathrm{H}), 1.91-1.84(\mathrm{~m}, 1 \mathrm{H}), 1.74-1.69(\mathrm{~m}, 2 \mathrm{H}), 1.64-1.58(\mathrm{~m}, 1 \mathrm{H}), 1.38-1.33(\mathrm{~m}, 1 \mathrm{H}), 1.22(\mathrm{~d}, J$ $=2.0 \mathrm{~Hz}, 3 \mathrm{H}), 1.07(\mathrm{~d}, J=2.0 \mathrm{~Hz}, 3 \mathrm{H}), 1.04(\mathrm{~s}, 3 \mathrm{H}), 1.00(\mathrm{~s}, 3 \mathrm{H}) ;{ }^{13} \mathrm{C} \mathrm{NMR}\left(125 \mathrm{MHz}, \mathrm{CDCl}_{3}\right) \delta$ 149.6, 148.4, 143.2, 140.0, 136.7, 132.0, 130.9, 121.8, 70.6, 60.9, 60.9, 35.4, 34.5, 32.0, 28.7, 28.3, 27.7, 27.1, 26.8, 26.8, 23.9, 23.8; IR (film) $\lambda_{\max } 3440,2959,1051,732 \mathrm{~cm}^{-1}$; HRMS (EI) calcd for $[\mathrm{M}]^{+}: \mathrm{m} / \mathrm{z}, 344.2351$, found 344.2344 .

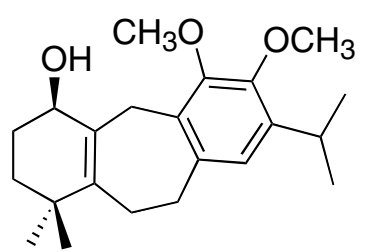

$(1 R)-9$

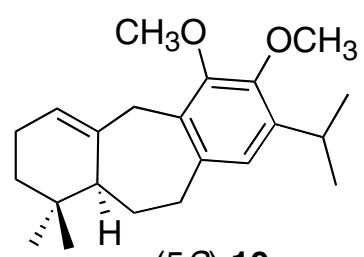

$(5 S)-10$

To a solution of tripheylphosphine $(1.50 \mathrm{~g}, 5.72 \mathrm{mmol})$ in $7.0 \mathrm{~mL}$ of anhydrous $N$-methyl morpholine at 0 ${ }^{\circ} \mathrm{C}$ was added DEAD $(1.1 \mathrm{~mL}, 6.95 \mathrm{mmol})$. The resulting mixture was stirred at $0{ }^{\circ} \mathrm{C}$ for $3-5$ minutes and cooled to $-30{ }^{\circ} \mathrm{C}$. Allylic alcohol $(1 R)-9(700 \mathrm{mg}, 2.04 \mathrm{mmol})$ in $7 \mathrm{~mL}$ of THF was added and the resulting mixture was stirred at $-30^{\circ} \mathrm{C}$ for another $30 \mathrm{~min}$. $o$-Nitrobenzenesulfonylhydrazine $(1.62 \mathrm{~g}, 7.50 \mathrm{mmol})$ was added to the reaction mixture before it solidified, followed by $5 \mathrm{~mL}$ of THF. The reaction mixture was vigorously shaken to dissolve any solid and then kept at $-30{ }^{\circ} \mathrm{C}$ for $3 \mathrm{~h}$. The temperature of the reaction was then raised to $30{ }^{\circ} \mathrm{C}$. After stirring at $30{ }^{\circ} \mathrm{C}$ for $2 \mathrm{~h}$, the reaction mixture was diluted by $30 \mathrm{~mL}$ of ethyl ether and cooled to $0{ }^{\circ} \mathrm{C}$. A solution of $5 \% \mathrm{H}_{2} \mathrm{O}_{2}(10 \mathrm{~mL})$ was added to the mixture and stirred at $0{ }^{\circ} \mathrm{C}$ for an additional hour. The reaction mixture was then passed through a column containing a one-inch wad of silica gel prior to a standard ethereal workup. The organic layer was then concentrated and the resulting light yellow oil was purified by silica gel chromatography (elution with pet ether/EtOAc $=20: 1)$ to give $360 \mathrm{mg}(54 \%)$ of $(5 S)-10$ as a white solid $\left[\mathrm{R}_{\mathrm{f}}=0.83\right.$, EtOAc/pet ether $\left.=1: 8\right]: \quad \mathrm{mp}=63-65^{\circ} \mathrm{C} ; \quad[\alpha]_{\mathrm{D}}=-73.10(\mathrm{MeOH} ; c 0.013)$; ${ }^{1} \mathrm{H}$ NMR (500 MHz, $\left.\mathrm{CDCl}_{3}\right) \delta 6.74(\mathrm{~s}, 1 \mathrm{H}), 5.53(\mathrm{~m}, 1 \mathrm{H}), 3.88(\mathrm{~s}, 3 \mathrm{H}), 3.86(\mathrm{~s}, 3 \mathrm{H}), 3.84(\mathrm{~d}, J=14.5 \mathrm{~Hz}, 1 \mathrm{H})$, 3.31 (septet, $J=6.5 \mathrm{~Hz}, 1 \mathrm{H}$ ), 3.07 (d, $J=14.5 \mathrm{~Hz}, 1 \mathrm{H}), 2.90-2.77$ (m, 2H), 2.09-2.03 (m, 2H), 1.97-1.91 (m, 1H), $1.86-1.80(\mathrm{~m}, 1 \mathrm{H}), 1.42-1.34(\mathrm{~m}, 1 \mathrm{H}), 1.25(\mathrm{~d}, J=2.0 \mathrm{~Hz}, 3 \mathrm{H}), 1.24(\mathrm{~d}, J=2.0 \mathrm{~Hz}, 3 \mathrm{H}), 1.22-1.19(\mathrm{~m}, 1 \mathrm{H})$, $1.16-1.10(\mathrm{~m}, 1 \mathrm{H}), 0.96(\mathrm{~s}, 3 \mathrm{H}), 0.92(\mathrm{~s}, 3 \mathrm{H}),{ }^{13} \mathrm{C} \mathrm{NMR}\left(125 \mathrm{MHz}, \mathrm{CDCl}_{3}\right) \delta 149.7,148.9,139.4,138.6,138.1$, 132.6, 121.8, 121.5, 60.9, 60.8, 51.7, 35.5, 35.3, 32.3, 31.6, 30.3, 27.8, 27.4, 26.9, 24.0, 23.8, 23.5; IR(film) $\lambda_{\max } 2957,1447,1410,1047,1000 \mathrm{~cm}^{-1}$; HRMS (EI) calcd for [M] ${ }^{+}: \mathrm{m} / \mathrm{z} 328.2402$, found 328.2392 . 


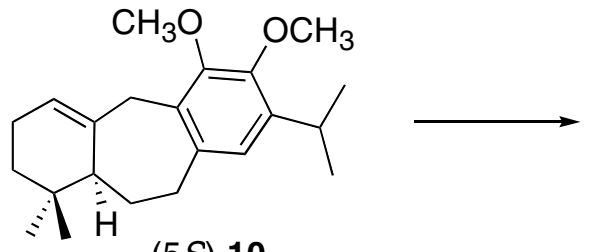

$(5 S)-10$

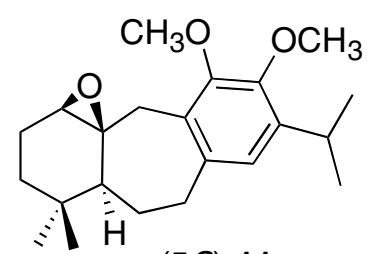

$(5 S)-11$

To a solution of $(5 S)-\mathbf{1 0}(222 \mathrm{mg}, 0.68 \mathrm{mmol})$ and in $\mathrm{CH}_{2} \mathrm{Cl}_{2}(10 \mathrm{~mL})$ at $0{ }^{\circ} \mathrm{C}$, was added $m$-CPBA $(0.343 \mathrm{~g}$ of a $77 \%$ mixture, $1.53 \mathrm{mmol}$ ). The resulting mixture was stirred for $2 \mathrm{~h}$ at $0{ }^{\circ} \mathrm{C}$ and quenched by $5 \% \mathrm{NaOH}$ solution $(3 \mathrm{~mL})$. Standard ethereal workup, followed by silica gel chromatography (elution with pet ether/Et ${ }_{2} \mathrm{O}$ $=10: 1)$, gave $221 \mathrm{mg}$ of $(5 S)-11(95 \%)$ as colorless oil $\left[\mathrm{R}_{\mathrm{f}}=0.67, \mathrm{EtOAc} /\right.$ pet ether $\left.=8: 1\right] . \quad[\alpha]_{\mathrm{D}}=-12.0$ $(\mathrm{MeOH} ; c 0.033) ; \quad{ }^{1} \mathrm{H}$ NMR $\left(500 \mathrm{MHz}, \mathrm{CDCl}_{3}\right) \delta 6.76(\mathrm{~s}, 1 \mathrm{H}), 3.84(\mathrm{~s}, 3 \mathrm{H}), 3.77(\mathrm{~s}, 3 \mathrm{H}), 3.37$ (d, $J=16.5 \mathrm{~Hz}$, $1 \mathrm{H}), 3.29$ (septet, $J=7.0 \mathrm{~Hz}, 1 \mathrm{H}), 3.23(\mathrm{~m}, 1 \mathrm{H}), 3.16-3.08(\mathrm{~m}, 1 \mathrm{H}), 2.70-2.62(\mathrm{~m}, 2 \mathrm{H}), 2.98-2.90(\mathrm{~m}, 2 \mathrm{H})$, $1.87-1.80(\mathrm{~m}, 1 \mathrm{H}), 1.69-1.54(\mathrm{~m}, 2 \mathrm{H}), 1.42-1.36(\mathrm{~m}, 1 \mathrm{H}), 1.26-1.20(\mathrm{~m}, 6 \mathrm{H}), 1.06-1.00(\mathrm{~m}, 1 \mathrm{H}), 0.90(\mathrm{~s}, 3 \mathrm{H})$, $0.77(\mathrm{~s}, 3 \mathrm{H}) ;{ }^{13} \mathrm{C}$ NMR $\left(125 \mathrm{MHz}, \mathrm{CDCl}_{3}\right) \delta 151.2,148.8,140.2,137.3,128.5,122.2,61.7,60.9,60.4,60.3$, 45.4, 36.8, 33.9, 31.4, 31.2, 29.5, 26.9, 26.8, 24.7, 23.9, 23.7, 22.3; IR(film) $\lambda_{\max } 2958,2931,1448,1411$, $1299,1046,925 \mathrm{~cm}^{-1}$; HRMS (EI) calcd for $[\mathrm{M}]^{+}: \mathrm{m} / z$ 344.2351, found 344.2348.

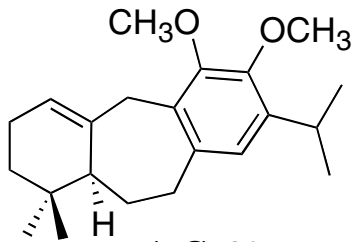

$(5 S)-10$

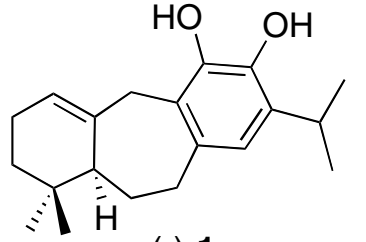

$(-)-1$

To a suspension of $\mathrm{NaH}(700 \mathrm{mg}, 60 \%, 17.5 \mathrm{mmol})$ in $\mathrm{DMF}(10 \mathrm{~mL})$ at $0{ }^{\circ} \mathrm{C}$, ethanethiol $(3.50 \mathrm{~mL}, 97 \%$, $45.8 \mathrm{mmol}$ ) was added dropwise. The resulting solution was cannulated into a suspension of $(5 S)-\mathbf{1 0}(318 \mathrm{mg}$, $0.97 \mathrm{mmol}$ ) in $2.0 \mathrm{~mL}$ of DMF. The reaction mixture was then stirred at $150{ }^{\circ} \mathrm{C}$ for $12 \mathrm{~h}$. After cooling to rt, the reaction mixture was diluted by $15 \mathrm{~mL}$ of ethyl ether, quenched by $3 \mathrm{~mL}$ brine, and acidified by $3 \mathrm{~mL} 1 \mathrm{~N}$ $\mathrm{HCl}$. The organic layer was washed with brine $(5 \times 5 \mathrm{~mL})$, dried over anhydrous $\mathrm{Na}_{2} \mathrm{SO}_{4}$, filtered and concentrated. Silica gel chromatography (elution with pet ether $/ \mathrm{Et}_{2} \mathrm{O}=4: 1$ ) gave $189 \mathrm{mg}(65 \%)$ of (-)-barbatusol (1) as a gray amorphous solid, which can be easily oxidized by air $\left[\mathrm{R}_{\mathrm{f}}=0.51\right.$, pet ether $/ \mathrm{EtOAc}=$ 2:1]: $[\alpha]_{\mathrm{D}}=-90.4\left(\mathrm{CHCl}_{3} ; c 0.044\right) ; \quad{ }^{1} \mathrm{H} \mathrm{NMR}\left(500 \mathrm{MHz}, \mathrm{CDCl}_{3}\right) \delta 6.53(\mathrm{~s}, 1 \mathrm{H}), 5.52(\mathrm{~s}, 1 \mathrm{H}), 5.14(\mathrm{br} \mathrm{s}, 2 \mathrm{H})$, 5.04 (br, 2H), $3.69(\mathrm{~d}, J=15.0 \mathrm{~Hz}, 1 \mathrm{H}), 3.16-3.04(\mathrm{~m}, 2 \mathrm{H}), 2.88-2.70(\mathrm{~m}, 2 \mathrm{H}), 2.08-1.98(\mathrm{~m}, 2 \mathrm{H}), 1.98-1.90$ (m. $1 \mathrm{H}), 1.85-1.78(\mathrm{~m}, 1 \mathrm{H}), 1.40-1.32(\mathrm{~m}, 1 \mathrm{H}), 1.30-1.24(\mathrm{~m}, 6 \mathrm{H}), 1.26-1.16(\mathrm{~m}, 1 \mathrm{H}), 1.12-1.08(\mathrm{~m}, 1 \mathrm{H}), 0.94(\mathrm{~s}$, $3 \mathrm{H}), 0.89$ (s, 3H); ${ }^{13} \mathrm{C}$ NMR (125 MHz, $\left.\mathrm{CDCl}_{3}\right) \delta 140.6,139.3,138.3,134.8,131.4,124.7,121.4,117.8,50.9$, $35.5,34.7,32.3,31.4,30.7,27.7,27.4,27.3,23.4,23.0,22.8$. 


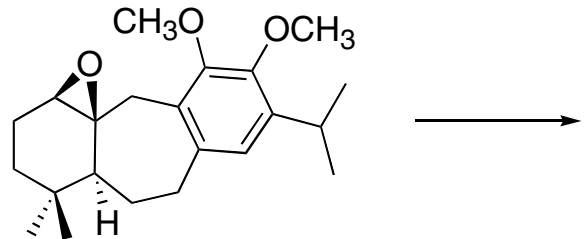

$(5 S)-11$

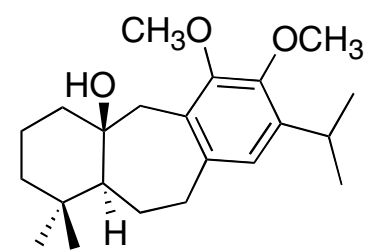

(10S)-12

To a solution of $(5 S)-\mathbf{1 1}(122 \mathrm{mg}, 0.35 \mathrm{mmol})$ in THF $(6.0 \mathrm{~mL})$ at $0{ }^{\circ} \mathrm{C}$, was added LAH $(28 \mathrm{mg}, 0.74$ mmol). The resulting mixture was refluxed for $20 \mathrm{~h}$ and then quenched at $0{ }^{\circ} \mathrm{C}$ with brine. Standard ethereal workup, followed by silica gel chromatography (elution with pet ether $/ \mathrm{Et}_{2} \mathrm{O}=10: 1$ ), gave $110 \mathrm{mg}$ of (10S)-12 $(92 \%)$ as a white solid $\left[\mathrm{R}_{\mathrm{f}}=0.67\right.$, pet ether/EtOAc $\left.=8: 1\right]: \quad \mathrm{mp}=130-133^{\circ} \mathrm{C} ; \quad[\alpha]_{\mathrm{D}}=8.50\left(\mathrm{CHCl}_{3} ; c 0.018\right)$; ${ }^{1} \mathrm{H}$ NMR (500 MHz, CDCl $\left.{ }_{3}\right) \delta 6.75(\mathrm{~s}, 1 \mathrm{H}), 3.83(\mathrm{~s}, 6 \mathrm{H}), 3.32-3.22(\mathrm{~m}, 2 \mathrm{H}), 2.82-2.64(\mathrm{~m}, 2 \mathrm{H}), 2.51(\mathrm{~d}, J=14.0$ $\mathrm{Hz}, 1 \mathrm{H}), 2.02-1.80(\mathrm{~m}, 3 \mathrm{H}), 1.60-1.51(\mathrm{~m}, 1 \mathrm{H}), 1.50-1.40(\mathrm{~m}, 2 \mathrm{H}), 1.36-1.24(\mathrm{~m}, 3 \mathrm{H}), 1.24-1.20(\mathrm{~m}, 6 \mathrm{H}), 0.94$ $(\mathrm{s}, 3 \mathrm{H}), 0.90(\mathrm{~s}, 1 \mathrm{H}) ;{ }^{13} \mathrm{C} \mathrm{NMR}\left(125 \mathrm{MHz}, \mathrm{CDCl}_{3}\right) \delta 152.5,149.0,140.9,140.3,127.6,121.2,70.9,60.9,58.5$, 42.7, 42.4, 41.6, 36.6, 34.6, 32.7, 26.9, 24.2, 24.0, 23.7, 21.8, 19.0; IR(film) $\lambda_{\max } 3471,2931,1451,1412,1050$ $\mathrm{cm}^{-1} ; \quad$ HRMS (EI) calcd for $[\mathrm{M}]^{+}: \quad \mathrm{m} / z$ 346.2508, found 346.2501.

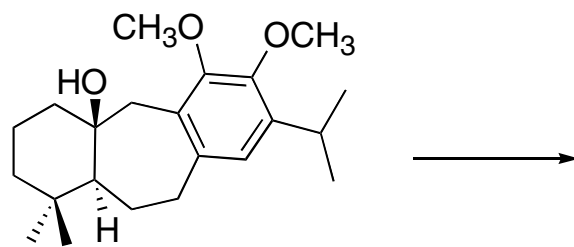

(10S)-12

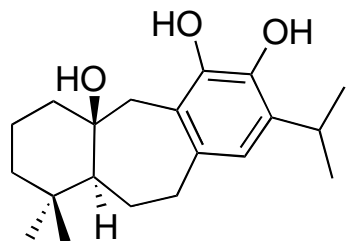

$(+)-2$

To a suspension of $\mathrm{NaH}(350 \mathrm{mg}, 60 \%, 8.75 \mathrm{mmol})$ in DMF $(6 \mathrm{~mL})$ at $0{ }^{\circ} \mathrm{C}$, ethanethiol $(1.40 \mathrm{~mL}, 97 \%$, $18.3 \mathrm{mmol}$ ) was added dropwise. The resulting solution was cannulated into a suspension of (10S)-12 (250 mg, $0.72 \mathrm{mmol}$ ) in $2.0 \mathrm{~mL}$ of DMF. The reaction mixture was then stirred at $160{ }^{\circ} \mathrm{C}$ for $12 \mathrm{~h}$. After cooling to rt, the reaction mixture was diluted by $15 \mathrm{~mL}$ of ethyl ether, quenched by $3 \mathrm{~mL}$ brine and acidified by $3 \mathrm{~mL} 1 \mathrm{~N} \mathrm{HCl}$. The organic layer was washed with brine $(5 \times 5 \mathrm{~mL})$, dried over anhydrous $\mathrm{Na}_{2} \mathrm{SO}_{4}$, filtered and concentrated. Silica gel chromatography (elution with pet ether/ $\left.\mathrm{Et}_{2} \mathrm{O}=4: 1\right)$ gave $161 \mathrm{mg}(70 \%)$ of (+)-demethylsalvicanol (2) as a white amorphous solid which can be easily oxidized by air $\left[\mathrm{R}_{\mathrm{f}}=0.42\right.$, pet ether/EtOAc $\left.=4: 1\right]$ : ${ }^{1} \mathrm{H}$ NMR $\left(500 \mathrm{MHz}, \mathrm{CDCl}_{3}\right) \delta 6.74$ (br s, 1H), $6.58(\mathrm{~s}, 1 \mathrm{H}), 5.90$ (br s, $\left.1 \mathrm{H}\right), 3.19$ (septet, $J=7.0 \mathrm{~Hz}, 1 \mathrm{H}$ ), 3.06 (d, $J=14.5$ $\mathrm{Hz}, 1 \mathrm{H}), 2.80-2.60(\mathrm{~m}, 2 \mathrm{H}), 2.56(\mathrm{~d}, J=14.5 \mathrm{~Hz}, 1 \mathrm{H}), 2.04-1.94(\mathrm{~m}, 1 \mathrm{H}), 1.90-1.72(\mathrm{~m}, 3 \mathrm{H}), 1.55-1.49(\mathrm{~m}, 1 \mathrm{H})$, $1.25(\mathrm{~d}, J=6.5 \mathrm{~Hz}, 3 \mathrm{H}), 1.22(\mathrm{~d}, J=6.5 \mathrm{~Hz}, 3 \mathrm{H}), 1.20-1.10(\mathrm{~m}, 1 \mathrm{H}), 0.93(\mathrm{~s}, 3 \mathrm{H}), 0.85(\mathrm{~s}, 3 \mathrm{H}) ;{ }^{13} \mathrm{C} \mathrm{NMR}$ $\left(125 \mathrm{M} \mathrm{Hz} \mathrm{CDCl}_{3}\right) \delta 142.9,141.4,136.5,133.2,121.4,118.1,72.1,58.3,42.5,41.8,41.5,36.3,34.6,32.4,27.3$, $24.3,22.8,21.8,21.7,18.8$. 


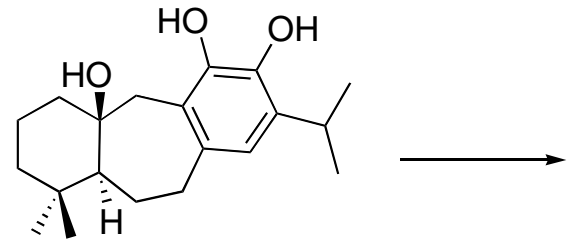

$(+)-2$

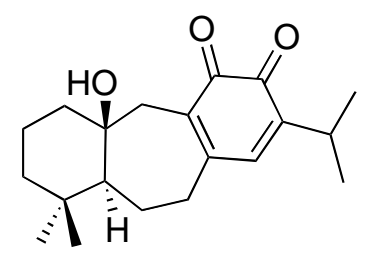

$(10 S)-13$

To a solution of (+)-demethylsalvicanol (2) (130 mg, $0.41 \mathrm{mmol})$ in ethyl ether ( $5 \mathrm{~mL})$ was added $\mathrm{Ag}_{2} \mathrm{CO}_{3}$ $(0.338 \mathrm{~g}, 1.22 \mathrm{mmol})$. The resulting mixture was stirred at $\mathrm{rt}$ for one hour before the reaction mixture was filtered through a column containing a one-inch wad of celite. The mixture was then concentrated to give 127 $\mathrm{mg}$ of quione $13(98 \%)$ as a dark red amorphous solid $\left[\mathrm{R}_{\mathrm{f}}=0.61\right.$, pet ether/EtOAc $\left.=4: 1\right]: \quad{ }^{1} \mathrm{H} \mathrm{NMR}(500 \mathrm{MHz}$, $\left.\mathrm{CDCl}_{3}\right) \delta 6.60(\mathrm{~s}, 1 \mathrm{H}), 3.70(\mathrm{~m}, 1 \mathrm{H}), 3.06(\mathrm{~d}, J=9.6 \mathrm{~Hz}, 1 \mathrm{H}), 2.93$ (septet, $\left.J=6.8 \mathrm{~Hz}, 1 \mathrm{H}\right), 2.67-2.42(\mathrm{~m}, 2 \mathrm{H})$, $2.18(\mathrm{~d}, J=6.8 \mathrm{~Hz}, 1 \mathrm{H}), 1.92-1.68(\mathrm{~m}, 3 \mathrm{H}), 1.62-1.48(\mathrm{~m}, 2 \mathrm{H}), 1.45-1.38(\mathrm{~m}, 2 \mathrm{H}), 1.31-1.20(\mathrm{~m}, 2 \mathrm{H}), 1.14-1.06$ (m, 6H), 0.93 (s, 3H), $0.92(\mathrm{~s}, 3 \mathrm{H}) ;{ }^{13} \mathrm{C} \mathrm{NMR}\left(125 \mathrm{MHz}, \mathrm{CDCl}_{3}\right) \delta 180.7,180.5,154.5,147.0,138.7,134.6$, $70.8,62.2,58.3,42.7,42.5,40.2,34.6,33.9,32.3,29.4,27.4,21.8,21.1,18.5$.

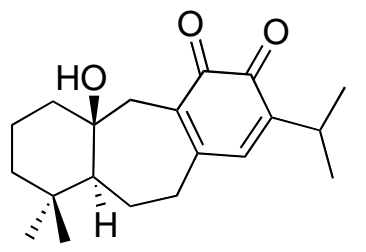

(10S)-13<smiles>CC(C)c1cc2c(c(O)c1O)C[C@@]13CCC[C@@H](C)[C@H]1C[C@H](O2)O3</smiles>

$(-)-3$

A concentrated solution of crude $(10 S)-\mathbf{1 3}(113 \mathrm{mg}, 0.36 \mathrm{mmol})$ in ethyl ether $(10 \mu \mathrm{L})$ was heated in a sealed $1 \mathrm{~mL}$ round bottom flask at $60{ }^{\circ} \mathrm{C}$ for $40 \mathrm{~h}$. Silica gel chromatography (elution with pet ether/ $\mathrm{Et}_{2} \mathrm{O}=4: 1$ ) of the resulting mixture gave $77 \mathrm{mg}(70 \%)$ of (-)-brussonol (3) as dark brown gum $\left[\mathrm{R}_{\mathrm{f}}=0.46\right.$, pet ether/EtOAc $=2: 1]: \quad[\alpha]_{\mathrm{D}}=-33.4\left(\mathrm{CHCl}_{3} ; c 0.024\right):{ }^{1} \mathrm{H} \mathrm{NMR}\left(400 \mathrm{MHz}, \mathrm{CDCl}_{3}\right) \delta 6.45(\mathrm{~s}, 1 \mathrm{H}), 5.26(\mathrm{br} \mathrm{s}, 1 \mathrm{H}), 5.17(\mathrm{br} \mathrm{s}$, $1 \mathrm{H}), 4.86(\mathrm{~d}, J=6.4 \mathrm{~Hz}, 1 \mathrm{H}), 2.73(\mathrm{~d}, J=16.4 \mathrm{~Hz}, 1 \mathrm{H}), 2.40(\mathrm{~d}, J=16.0 \mathrm{~Hz}, 1 \mathrm{H}), 2.16-2.08(\mathrm{~m}, 1 \mathrm{H}), 2.03-1.94$ $(\mathrm{m}, 1 \mathrm{H}), 1.93-1.86(\mathrm{~m}, 1 \mathrm{H}), 1.83-1.76(\mathrm{~m}, 3 \mathrm{H}), 1.64-1.47(\mathrm{~m}, 2 \mathrm{H}), 1.24(\mathrm{~d}, J=3.2 \mathrm{~Hz}, 3 \mathrm{H}), 1.22(\mathrm{~d}, J=3.2 \mathrm{~Hz}$, $3 \mathrm{H}), 1.20-1.08(\mathrm{~m}, 1 \mathrm{H}), 0.96(\mathrm{~s}, 3 \mathrm{H}), 0.84(\mathrm{~s}, 3 \mathrm{H}) ;{ }^{13} \mathrm{C} \mathrm{NMR}\left(125 \mathrm{MHz}, \mathrm{CDCl}_{3}\right) \delta 141.8,139.7,134.4,132.2$, 116.7, 112.9, 80.3, 79.4, 51.3, 39.9, 39.0, 32.4, 32.1, 30.9, 30.8, 27.3, 26.9, 23.0, 22.8, 16.4 .

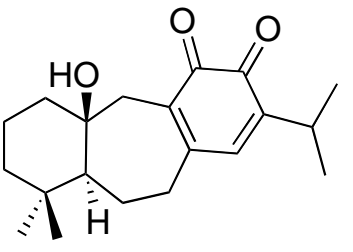

(10S)-13

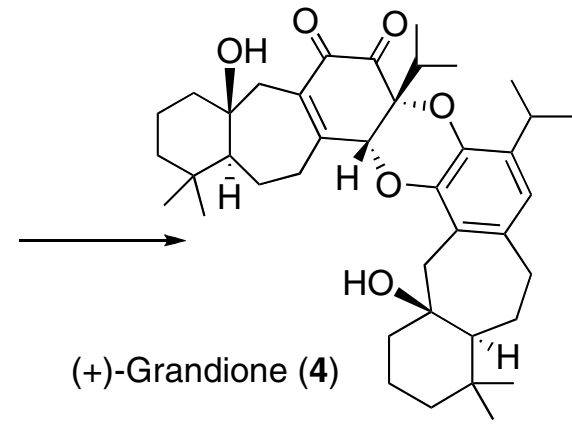


Crude quinone $13(130 \mathrm{mg}, 0.41 \mathrm{mmol})$ was heated in a sealed $3 \mathrm{~mL}$ conical vile flask at $50{ }^{\circ} \mathrm{C}$ for $60 \mathrm{~h}$. Silica gel chromatography (elution with pet ether/ $\mathrm{Et}_{2} \mathrm{O}=6: 1$ ) of the resulting mixture gave $93.7 \mathrm{mg}(72 \%)$ of (+)-grandione (4) as yellow needles $\left[\mathrm{R}_{\mathrm{f}}=0.55\right.$, pet ether/EtOAc $\left.=4: 1\right]: \quad[\alpha]_{\mathrm{D}}=+66\left(\mathrm{CHCl}_{3} ; c 0.033\right) ;{ }^{1} \mathrm{H}$ $\operatorname{NMR}\left(500 \mathrm{MHz}, \mathrm{CDCl}_{3}\right) \delta 6.65(\mathrm{~s}, 1 \mathrm{H}), 4.60(\mathrm{~s}, 1 \mathrm{H}), 3.28$ (septet, $\left.J=6.5 \mathrm{~Hz}, 1 \mathrm{H}\right), 3.10(\mathrm{~d}, J=14.5 \mathrm{~Hz}, 1 \mathrm{H})$, 2.86-2.70 (m, 3H), 2.67-2.58 (m, 2H), $2.36(\mathrm{~d}, J=14.0 \mathrm{~Hz}, 1 \mathrm{H}), 2.28(\mathrm{~d}, J=15.0 \mathrm{~Hz}, 1 \mathrm{H}), 2.00-1.84(\mathrm{~m}, 2 \mathrm{H})$, $1.86-1.68(\mathrm{~m}, 4 \mathrm{H}), 1.68-1.60(\mathrm{~m}, 2 \mathrm{H}), 1.60-1.52(\mathrm{~m}, 4 \mathrm{H}), 1.52-1.39(\mathrm{~m}, 5 \mathrm{H}), 1.36-1.31(\mathrm{~m}, 1 \mathrm{H}), 1.30(\mathrm{~d}, J=1.5$ $\mathrm{Hz}, 3 \mathrm{H}), 1.29(\mathrm{~d}, J=1.5 \mathrm{~Hz}, 3 \mathrm{H}), 1.28-1.20(\mathrm{~m}, 5 \mathrm{H}), 1.11(\mathrm{~d}, J=7.0 \mathrm{~Hz}, 3 \mathrm{H}), 1.01(\mathrm{~d}, J=7.0 \mathrm{~Hz}, 3 \mathrm{H}), 0.94$ (s, 3H), 0.92 (s, 3H), 0.88 (s, 3H); ${ }^{13} \mathrm{C}$ NMR (125 MHz, $\left.\mathrm{CDCl}_{3}\right) \delta 191.9,186.5,159.7,141.4,141.0,137.9,137.1$, 134.9, 121.6, 119.5, 86.4, 78.2, 71.1, 71.0, 58.3, 58.2, 43.0, 42.7, 42.4, 40.4, 40.1, 36.1, 34.6, 34.5, 32.4, 32.3, $30.7,27.8,24.3,22.7,22.1,21.8,21.8,20.4,19.1,18.4,16.9,16.3$.

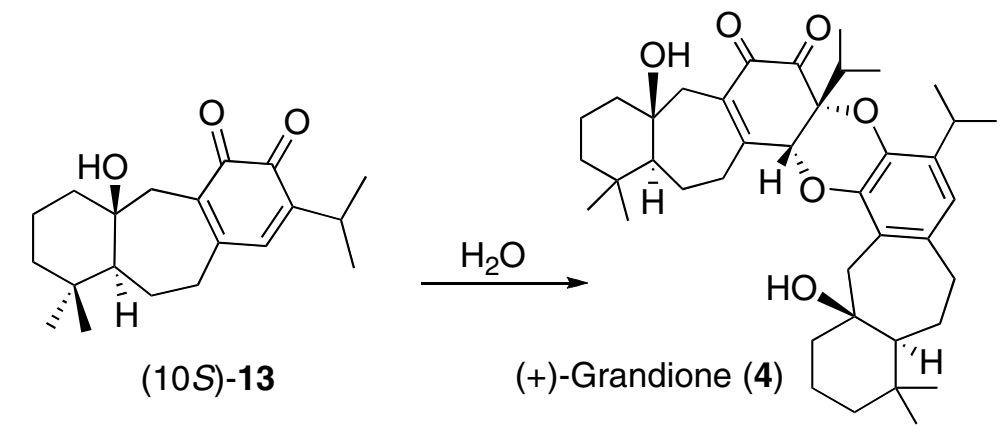

Crude quinone $13(111 \mathrm{mg}, 0.350 \mathrm{mmol})$ and $3 \mathrm{~mL}$ of water were heated in a sealed $5 \mathrm{~mL}$ mico-reaction vial at $50{ }^{\circ} \mathrm{C}$ for $60 \mathrm{~h}$. After standard ethereal workup, a ${ }^{1} \mathrm{H}$ NMR indicated that the crude reaction residue consisted of a 3:1 mixture of grandione and brussonol, respectively. Silica gel chromatography (elution with pet ether/Et ${ }_{2} \mathrm{O}=6: 1$ ) gave recovered 13 and $58 \mathrm{mg}(53 \%)$ of grandione (4) and $9 \mathrm{mg}(8 \%)$ of brussonol which were identical to that previously prepared. The yields cited are conversion yields. 